\title{
Association of Ankle-Brachial Index with the Angiographic Severity of Patient with Coronary Artery Disease
}

\author{
Abul Hasnat Md. Jafar, Md.Abdul Kader Akanda, Md. Khalequzzaman, Sanjib Chowdhury, \\ Muhammad Azmol Hossain, Sharadindu Shekhar Roy, Abeeda Tasnim Reza, Khondker \\ Rafiquzzaman, Ratan Kumar Datta, Md. Minhaj Arefin, Fathima Aaysha Cader. \\ Department Of Cardiology, National Institute of Cardiovascular Diseases, Dhaka
}

Keywords:

Ischaemic heart disease, Peripheral vascular disease, Ankle brachial index.

\begin{abstract}
:
Background: The Ankle-Brachial Index has been shown to be a good marker of systemic atherosclerosis and a powerful indicator of cardiovascular morbidity and mortality This study evaluated the relation of $A B I$ with the angiographic severity of patient with coronary artery disease.

Methods: This is a hospital based cross-sectional analytical study. 100 adult Bangladeshi patients who were admitted and underwent coronary angiography according to inclusion and exclusion criteria were the study population. All patient's ABI were measured and coronary angiography were done. Patients were divided into two groups according to ABI. Group I with $A B I>0.90$ and group II with $A B I<0.90$.

Results: In group I 65(82.2\%) were men and 16(19.8\%) were women. In group II 18(94.7\%) patients were men and $1(5.3 \%)$ was women. Significant co-relation was found between low ABI and severity of CAD. Low $A B I$ group showed more severe form of $C A D$ with higher prevalence of triple vessel diseases, significant stenosis and more involvement of left main (LM) and left anterior descending(LAD)artery. Single vessel disease was found more with normal ABI. Hypertension, diabetes mellitus and Low ABI showed predictors of significant severe stenosis of coronary arteries.

Conclusion: Low ankle brachial index is a predictor of the severity of coronary artery disease. So it could be incorporated in our day to day clinical cardiology practice as non-invasive, bedside test to assess and predict the severity of coronary artery disease.
\end{abstract}

(Cardiovasc. j. 2018; 10(2): 201-205)

\section{Introduction:}

Cardiovascular diseases (CVD) account for more than 17 million deaths globally each year. It contributes to $30 \%$ of all deaths, $80 \%$ of which occur in low-income and middle-income countries, and this figure is expected to grow to 23.6 million by 2030 . Ischemic heart disease (IHD) alone caused 7 million deaths worldwide in 2010, an increase of $35 \%$ since $1990 .{ }^{1}$ The exact prevalence of coronary artery disease (CAD) in Bangladesh is not known. Only a limited number of small-scale epidemiological studies are available. Probably the prevalence of IHD was first reported in 1976, which was $0.33 \% .^{2}$ More recent data indicates $\mathrm{CAD}$ prevalence between $1.85 \%$ and $3.4 \%$ in rural and $19.6 \%$ in an urban sample of working professionals. ${ }^{3}$

Atherosclerotic changes in the arterial wall progress slowly and quietly after starting in the early stages of life. But the clinical manifestation usually appears at the advanced stage. ${ }^{4}$ Since atherosclerosis is a systemic disease. There is more than one arterial territory affected at the same time in $12-40 \%$ cases. ${ }^{5}$ Several epidemiological studies have shown positive association between $\mathrm{CAD}$ and peripheral artery disease (PAD). ${ }^{5}$

Ankle-brachial index (ABI), also named as Anklebrachial pressure index (ABPI), used to diagnose PAD has been shown to be a good marker of systemic atherosclerosis and a powerful indicator of cardiovascular morbidity and mortality. PAD is highly prevalent among patient with angiographically documented CAD. Studies showed that about $29 \%$ patient of PAD are asymptomatic, who can be identified by ABI. ABI is a simple, easy, non-invasive, inexpensive bedside clinical test used in the diagnosis of $\mathrm{PAD}$

Address of Correspondence: Dr. Abul Hasnat Md. Jafar, Department of Cardiology, National Institute of Cardiovascular Diseases, Dhaka, Bangladesh. 
and can be incorporated to daily clinical practice to predict the severity of CAD and can identify individuals at risk for cardiovascular disease in other arteries of the body, especially the coronary and carotid arteries. The normal range of $\mathrm{ABI}$ is 0.9 to 1.4 .It has $90 \%$ sensitivity and $98 \%$ specificity in detecting $>50 \%$ stenosis in lower limb arteries associated with an increased risk of myocardial infarction and stroke. ${ }^{4}$ Aim of this study was to see the association of ABI with the severity of Coronary artery disease in our population of Bangladesh.

\section{Methods:}

This cross sectional analytical study was conducted in the Department of cardiology, National Institute of Cardiovascular Diseases, Dhaka, Bangladesh from April 2016 to March 2017, Patients with chronic stable angina (CSA), unstable angina (UA), Non ST elevation myocardial infarction (NSTEMI) and ST elevation myocardial infarction (STEMI) undergoing coronary angiogram were included in the study. Patients with prior PCI or CABG, valvular or congenital heart diseases, congenital vascular diseases, established malignancy, severe lung, liver or renal co-morbidities were excluded from the study. Informed written consent was taken from each patient before enrollment and meticulous history was taken.

Demographic data such as, age, sex, were recorded. Risk factors profile including smoking, hypertension, diabetes mellitus, obesity, dyslipidemia and family history of coronary artery disease were noted. All peripheral pulses and precordium were examined. Laboratory investigations were done such as serum creatinine, fasting blood sugar\& fasting Lipid profile through standard laboratory techniques.12 lead resting ECG was done and transthoracic echocardiography was done before coronary angiogram.

ABI of all study patients was measured. The measurement was done under the supervision of consultant of vascular surgery by a Multi Doppler II Advanced Bidirectional Doppler (Huntleigh, UK0. On the basis of ABI results, the patients were divided into two groups. Those with ABI $>0.9$ were in Group- I and those with $<0.9$ were in Group- II. Coronary angiogram was done by conventional method. Severity of coronary stenosis was graded according to Gensini score. ${ }^{6}$

\section{Results:}

A total of 100 patients with CAD who agreed to undergo coronary angiography were enrolled in the study. Coronary angiogram was done during index hospital admission. The mean age of group I was $53.5 \pm 8.2$ years, the mean age of group II was $57.7 \pm 7.4$ years, The mean age of group II was more than group I patients and the age difference between two groups was statistically significant $(p=0.04)$. Male patients were predominant in both groups. But it was not statistically significant $(\mathrm{p}=0.18)$,

Regarding ricks factors, it was observed that highest percentage had history of dyslipidaemia (79\%) followed by smoker (55.6\%), hypertension (49.4\%), diabetes mellitus (46.9\%), and family history of CAD 29.6\% in Group I patients. In group II patients, highest percentage had dyslipidaemia (84.2\%) followed by hypertension and diabetes mellitus (73.7\%), smoker and family history of CAD (52.6\%).

STEMI was present higher in group II compared to group I (42.0\% vs. $18.5 \%)$ with statistically significant association $(p=0.02)$. NSTEMI was present higher percentages in group I than group II $(28.4 \%$ vs. $21.1 \%)$ with statistically insignificant association $(\mathrm{p}=0.51)$. UA was present higher in group I than group II $(27.2 \%$ vs. $15.8 \%$ ) with statistically insignificant association $(p=0.30)$. CSA had observed insignificantly higher in group I than group II (24.8\% vs. $10.5 \%$, p=0.17). MI with CHF was found higher in group II compared to group I (10.5\% vs. $1.2 \%)$ with significant association $(\mathrm{p}=0.03)$.

LM and LAD arteries were more frequently involved in group II compared to group I with statistically significant association $(p=0.04$, $\mathrm{p}=0.01$ ). LCX and RCA had also higher in group II than group I but failed to get statistical level of significance $(\mathrm{p}=0.17$, and 0.58$)$.

Mean Gensini Score was significantly higher in group II compared to group I $(72.7 \pm 33.1$ vs. $26.4 \pm 20.4, \mathrm{p}<.001)$. 
There was a negative correlation between ABI and coronary artery disease severity in terms of Gensini score ( $r=-0.65)$. It was observed that the Pearson's correlation is statistically significant $(p=<0.001)$ by correlation t-test. The figure indicated that $\mathrm{ABI}$ was decreasing as Gensini score was increasing.

There was a binary logistic regression analysis of odds ratio (OR) for characteristics of the subjects likely to cause coronary artery disease patients. Univariate and multivariate analysis revealed that out of the 7 variables hypertension, diabetes mellitus and low Ankle-Brachial Index (ABI) were found to be the independently significant predictors of severe CAD patients with ORs being 1.97 vs. $1.52,3.78$ vs. 4.61 and 10.39 vs. 9.77 respectively.

There was a negative correlation between ABI and coronary artery disease severity in terms of Gensini score ( $r=-0.65$. Pearson's correlation was statistically significant $(\mathrm{p}=<0.001)$ by correlation t-test. The figure indicated that ABI was decreasing as Gensini score was increasing.

Table-I

Baseline Clinical Characteristics of Study population. $(n=100)$.

\begin{tabular}{lccc}
\hline & Group I $(\mathrm{n}=81)$ & Group II $(\mathrm{n}=19)$ & p value \\
\hline Age in years (Mean \pm SD) & $53.5 \pm 8.2$ & $57.7 \pm 7.4$ & $0.04^{\mathrm{s}}$ \\
Smoker & $45(55 \% 6)$ & $10(52.6 \%)$ & 0.82 \\
Hypertensive & $40(49.4 \%)$ & $14(73.7 \%)$ & 0.04 \\
Diabetic & $38(46.9 \%)$ & $14(73.7 \%)$ & 0.03 \\
Dyslipdemic & $64(79 \%)$ & $16(84.2 \%)$ & 0.61 \\
Family H/O of CADPresent & $24(29.6 \%)$ & $10(52.6 \%)$ & 0.06 \\
BMI (Mean \pm SD) & $25.9 \pm 3.1$ & $27.6 \pm 2.3$ & 0.03 \\
STEMI & $15(18.5 \%)$ & $8(42 \%)$ & 0.02 \\
NSTEMI & $23(28.4 \%)$ & $4(21.1 \%)$ & 0.51 \\
UA & $22(27.2 \%)$ & $3(15.8 \%)$ & 0.30 \\
CSA & $20(24.8 \%)$ & $2(10.5 \%)$ & 0.17 \\
MI with CHF & $1(1.2 \%)$ & $2(10.5 \%)$ & 0.03 \\
LM & $0(0.0 \%)$ & $2(10.5 \%)$ & 0.04 \\
LAD & $40(49.4 \%)$ & $15(78.9 \%)$ & 0.01 \\
LCX & $33(40.7 \%)$ & $11(57.9 \%)$ & 0.17 \\
RCA & $37(45.7 \%)$ & $10(52.6 \%)$ & 0.58 \\
Gensini severe(>36 points) & $23(28.4 \%)$ & $15(79 \%)$ & $<0.001$ \\
Gensini Moderate to no disease (<36 points) & $58(71.6 \%)$ & $4(21.0 \%)$ & $<0.001$ \\
CAD severity (by Gensini Score)Mean \pm SD & $26.4 \pm 20.4 \%$ & $72.7 \pm 331 \%$ & $<0.001$ \\
\hline
\end{tabular}

LAD, Left, anterior descending artery, RCA- Right coronary artery, LCX-Left circumflex artery, UA-Unstable angina, NSTEMINon ST elevation MI, STEMI-ST elevation MI and CSA-Chronic stable angina.

Table-II

Univariate and multivariate binary logistic regression analysis of determinants of CAD (by Gensini Score).

\begin{tabular}{|c|c|c|c|c|}
\hline \multirow[t]{2}{*}{ Variables of interest } & \multicolumn{2}{|c|}{ Univariate } & \multicolumn{2}{|c|}{ Multivariate } \\
\hline & OR $(95 \%$ CI $)$ & $\mathrm{p}$ value & OR $(95 \% \mathrm{CI})$ & $\mathrm{p}$ value \\
\hline Advance age (>55yrs) & $0.94(0.420-2.100)$ & $0.88^{\mathrm{ns}}$ & $0.54(0.180-1.598$ & $0.26^{\mathrm{ns}}$ \\
\hline Smoking & $0.65(0.293-1.458)$ & $0.29^{\mathrm{ns}}$ & $0.64(0.211-1.980)$ & $0.44^{\mathrm{ns}}$ \\
\hline Hypertension & $1.97(1.201-4.173)$ & $0.02^{\mathrm{s}}$ & $1.52(1.171-3.534$ & $0.04^{\mathrm{s}}$ \\
\hline Dyslipidemia & $1.37(0.495-3.807)$ & $0.54^{\mathrm{ns}}$ & $0.88(0.181-4.318)$ & $0.87^{\mathrm{ns}}$ \\
\hline Diabetes mellitus & $3.78(1.613-8.870)$ & $0.002^{\mathrm{s}}$ & $4.61(1.249-16.604)$ & $0.02^{\mathrm{s}}$ \\
\hline Increased BMI & $1.13(1.009-3.298)$ & .04 & $0.99(0.829-1.93)$ & $0.64^{\mathrm{ns}}$ \\
\hline Low ABI & $10.39(3.723-14.998)$ & $0.003^{\mathrm{s}}$ & $9.77(2.425-16.998)$ & $0.001^{\mathrm{s}}$ \\
\hline
\end{tabular}




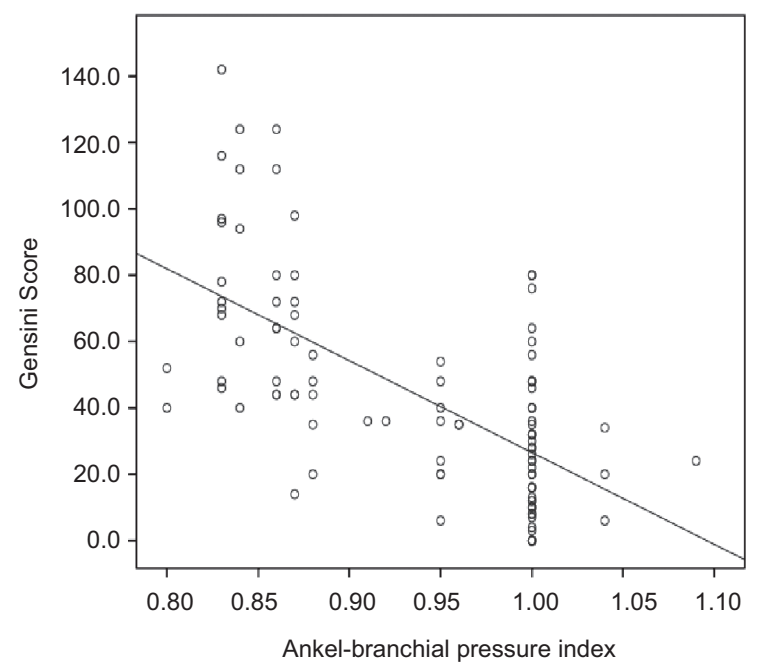

Fig.-1: Scatter plot diagram showing correlation between Gensini Score and ABI $(n=100)$.

\section{Discussion}

The mean age of the studied patient was $54.3 \pm 8.2$ years. Another similar study found mean age was $56 \pm 11$ in group-I \& $61 \pm 11$ in group-II which was close to present study. ${ }^{6}$ The mean age of group II was more than group I patients and the age difference between two groups was statistically significant in both studies $(\mathrm{p}=0.04$ $\& \mathrm{p}=<0.01$ respectively). One Study in Bangladesh found mean age was $56.63 \pm 8.95$ years. ${ }^{7}$

Among the studied patients, highest percentage had history of dyslipidaemia followed by smoking, hypertension, diabetes mellitus and family history of CAD in Group I patients. In group II patients, risk factors had like as highest percentage had dyslipidaemia followed by hypertension and diabetes mellitus, smoking and family history of CAD. It was found that hypertension and diabetes mellitus were significantly higher in group II than group I. The rest of the risk factors had no significant association between study groups. One Study in Bangladesh reported high prevalence of hypertension in elderly Bangladeshi population (40-65\%) to contribute the CAD. ${ }^{3}$ there are some differences regarding the risk factor profiles in different countries. ${ }^{4,6,8}$ These differences might be due to variation in the life style, degree of motivation and level of education.

Coronary artery disease (CAD) severity of the study patients were assessed by Gensini score. ${ }^{9}$ and score was done with $>36$ points as moderate to severe. ${ }^{10}$ It was found that moderate to severe form of CAD (Gensini scoree" 36 points) was $79.0 \%$ and $28.4 \%$ in group II and group I respectively. Moderate to no disease form of CAD (Gensini score <36) was found $21.0 \%$ and $71.6 \%$ in group II and group I respectively. Severe form of CAD patients were significantly more in group II patients with low ABI.

Other studies also found inverse relationship of $\mathrm{ABI}$ with the severity of $\mathrm{CAD}$ assessed by Gensini score which was compatible to our study. ${ }^{11}$ Another Study of Saudi Arabia found mean number of vessel involvement is higher $(2.10 \pm 0.736)$ in patients with $<0.90 .^{5}$

The mean level of Ankle-Brachial Index (ABI) was observed $0.99 \pm 0.03$ in group I and $0.85 \pm 0.02$ in group II which was statistically significant $(p<0.001)$ and was compatible with other studies. ${ }^{5,12}$ In our study STEMI and MI with CHF were more in low ABI which is consistent with other studies. ${ }^{5}$ Regarding vessel involvement, left main and LAD involvement were higher in group II $(\mathrm{ABI}<0.90)$ than in group I $(\mathrm{ABI}>0.90)$ which was also concordant other studies

Regarding the severity of CAD in terms of Gensini score, it was found that ABI had negative correlation with ABI which was statistically significant $(p=<0.001)$.ABI was decreasing as Gensini score was increasing. Same results were also found in other studies. ${ }^{5,11}$

This study demonstrated the binary logistic regression analysis of odds ratio (OR) for characteristics of the subjects likely to cause coronary artery disease patients. Univariate and multivariate analysis revealed that hypertension, diabetes mellitus, low Ankle-Brachial Index were found to be the independent significant predictors of severe CAD patients. These were concordant with other studies. Thus the ABI was found to be independent predictor of the severity of $\mathrm{CAD}$.

\section{Study Limitations:}

This study was done among a small number of patients. Sampling method was nonrandomized, conducted in a single center. Coronary angiography was assessed by visual observation. 


\section{Conclusion:}

Ankle-Brachial Index which was usually used to diagnose peripheral artery disease could also be used as a clinical test to predict severity of coronary artery disease. It was found as a predictor of the severity of Coronary Artery Disease. So it could be incorporated in our day to day clinical cardiology practice as non-invasive, bedside test to assess and predict the severity of coronary artery disease.

\section{Conflict of Interest - None.}

\section{References:}

1. Wong ND. Epidemiological studies of CHD and the evolution of preventive cardiology. Nature Reviews, Cardiology 2014; 11(5): 276-289.

2. Malik A, Islam MN, Zafor A, Khan AK, Ramizuddin M. Clinical patterens of ischemic heart diseases and its association with some known risk factors. Bangladesh Heart journal 1987; 2:11.

3. Islam AKM, Majumder AAS. Coronary artery disease in Bangladesh: A review. Indian Heart J 2013; 65(4): 424435.

4. Oliveira DCd, Correia A, Neto JN, Gurgel M, Sarinho FW. Victor EG. Association Between Ankle-Brachial Index and Coronary Lesions Assessed by Coronary Angiography. Cardiology Research 2015; 6(1): 216-220.
5. Alshehri AM, Elsharawy M. Importance of Ankle/Brachial Pressure Index in Saudi Patients with Coronary Artery Disease. World Journal of Cardiovascular Surgery 2013; 03: $181-181$.

6. Sabedotti M, Leite RS, Quadros ASD. Ankle-Brachial Index as a Predictor of Significant Coronary Artery Disease in Patient Undergoing Coronary Angiography. Rev Bras Cardiol Invasiva 2014; 22(4): 359-363.

7. Taimur SDM, Haque MM, Rashid MA, Ali SMK, Shahajahan M, Islam F. Association between Peripheral Arterial Disease and Coronary Artery Disease among Tobacco User Diabetic Patients. Bangladesh Heart Journal 2017; 32(1): 3-9.

8. Habib SMA, Islam MN, Pasha K, Alam SAN, Mohsin K, Islam KK, Ullah M, Khelequzzaman M, Siddique MA, Sultan MAU. Association of Ankle-Brachial Index with the angiographic severity of patient with coronary artery disease. University Heart Journal 2010; 6(1): .

9. Gensini GG. A more meaningful scoring system for determining the severity of coronary heart disease. Am J Cardiol1983; 51(3): 606-606.

10. Sun L, Lu SZ. Association between non-alcoholic fatty liver disease and coronary artery disease severity. Chinese Med J 2011; 124 (6): 867-872.

11. Saffar HBA, Ghizzi HJA, Muhammad SM. The AnkleBrachial Pressur Index as a Predictor of Significant Coronary Artery Disease Severity. J Fac Med Baghdad 2009; 51: 3.

12. Singal KK, Singal N, Gupta P, Ranpal JS. Bangladesh Journal of Medical Science Vol. 15 No. 